Atıf için / For Citation: F. Yildirim, " 3-Amino-5-Hidroksi-1H-Pirazol Bazlı Bazı Yeni Disazo Boyarmaddelerinin Sentezi ve Spektroskopik Özellikleri”, Süleyman Demirel Üniversitesi Fen Edebiyat Fakültesi Fen Dergisi, 16(1), 189-199, 2021.

\title{
3-Amino-5-Hidroksi-1H-Pirazol Bazlı Bazı Yeni Disazo Boyarmaddelerinin Sentezi ve Spektroskopik Özellikleri
}

\author{
Fati YILDIRIM $^{* 1}$ \\ ${ }^{1}$ Pamukkale Üniversitesi, Denizli Teknik Bilimler Meslek Yüksekokulu, Kimya ve Kimyasal İşleme \\ Teknolojileri Bölümü, Kimya Teknolojisi Programı, 20160, Denizli, Türkiye \\ *yazışllan yazar e-posta: fati@pau.edu.tr
}

(Alinış / Received: 01.03.2021, Kabul / Accepted: 04.05.2021, Yayımlanma / Published: 27.05.2021)

Özet: Bu çalışmada, yeni bir dizi 3-amino-5-hidroksi-1H-pirazol türevi boyarmadde sentezlendi ve karakterize edildi. Bu maksatla, $m$ - ve $o$ - sübstitüe anilin türevleri öncelikle diazoland, elde edilen bileşikler 3-amino krotononitril ile kenetlendi. Elde edilen ürün (1a-h) hidrazin monohidrat ile etkileştirilerek 5-amino-4-arilazo-3-metil-1H-pirazoller (2a-h) sentezlendi. Sonrasinda 5-amino-4-arilazo-3-metil-1H-pirazoller diazoland1 ve 3-amino-5-hidroksi-1Hpirazol ile kenetlenerek 8 adet yeni disazo boyarmaddesi (3a-h) sentezlenmiş oldu. Elde edilen sekiz boyarmaddenin yapısı Fourier dönüşümlü infrared spektroskopisi (FT-IR), Nükleer manyetik rezonans spektroskopisi ( $\left.{ }^{1} \mathrm{H}-\mathrm{NMR}\right)$, Ultraviyole görünür bölge absorpsiyon spektroskopisi (UV-Vis) ve element analizi gibi çeşitli spektrofotometrik yöntemlerle belirlendi. Alt1 farklı çözücüde boyarmaddelerin maksimum absorpsiyon dalga boyları belirlendi ve absorpsiyon spektrumları üzerine asit-baz etkisi incelendi. Bu çalışma sonunda tekstil ve azo boyarmadde literatürüne 8 adet yeni aminopirazol türevi disazo boyarmaddeleri kazandırıldı.

Anahtar kelimeler: Heterosiklik bileşikler, Disazo boyarmaddeler, Aminopirazol

\section{Synthesis and Spectroscopic Properties of Some New Disazo Dyes Based on 3-Amino-5-Hydroxy-1H-Pyrazole}

\begin{abstract}
In this study, a new series of 3-amino-5-hydroxy-1H-pyrazole derivative dyes were synthesized and characterized. For this purpose, firstly the $m$ - and $o$ - substituted aniline derivatives were diazotised, the resulting compounds were coupled with 3-amino crotononitrile. 5-amino-4-arylazo-3-methyl-1H-pyrazoles $(2 \mathrm{a}-\mathrm{h})$ were synthesized by reacting the obtained product (1a-h) with hydrazine monohydrate. Subsequently, the 5-amino-4-arylazo-3-methyl-1Hpyrazoles were diazotised and coupled with 3-amino-5-hydroxy-1H-pyrazole to synthesize 8 new disazo dyes (3a-h). The structure of the eight dyes obtained were determined by various spectrophotometric methods such as Fourier transform infrared spectroscopy (FT-IR), Nuclear magnetic resonance spectroscopy ( $\left.{ }^{1} \mathrm{H}-\mathrm{NMR}\right)$, Ultraviolet visible region absorption spectroscopy (UV-Vis) and elemental analysis. The maximum absorption wavelengths of the dyes in six different solvents were determined and the acid-base effects on the absorption spectra were investigated. At the end of this study, 8 new aminopyrazole derivative disazo dyes were introduced to the textile and azo dyestuff literature.
\end{abstract}

Key words: Heterocyclic compounds, Disazo dyes, Aminopyrazole 


\section{Giriș}

Yapılarında azo parçasının $(\mathrm{N}=\mathrm{N})$ varlığı ile karakterize edilen azo boyarmaddeleri, dispers boyaların en temel grubu olup [1] büyük bir sentetik organik bileşik sınıfını temsil eder [2] ve teknolojinin çeşitli alanlarındaki farklı uygulamaları nedeniyle büyük ilgi görmektedir. Azo boyarmaddelerin en önemli faydaları boyama mukavemeti, kolay üretim, geniş renk skalaları ve kaliteli renk haslığı gibi özelliklerinden dolayı renklendirme özellikleri olarak kalsada [3-12], ayrıca güneş pilleri, fotoduyarlılaştırıcılar, sensörler, fotokromik malzemeler, metalokromik göstergeler ve elektro-optik cihazlar [13-18] olarak da pek çok alanda kullanılmaktadır. Azo boyarmaddeler son zamanlarda birçok teorik çalışmanın konusu haline gelmiş $[19,20]$, biyoloji alanındaki araştırmacıların ilgisini çekmiş ve azo boyarmaddelerin antimikrobiyal özelliklere sahip olduğu [21,22] ve antifungal aktivite gösterdiği bildirilmiştir [23].

Pirazol halkaları beş üyeli olup iki bitişik nitrojen atomu içerir. Pirazol türevleri, yalnızca ticari boyaların imalatındaki uygulamaları nedeniyle değil, aynı zamanda potansiyel farmakolojik ve biyolojik uygulamaları nedeniyle de çok önemli bir bileşik sınıfı olarak kabul edilirki yapılan çalışmalarda pirazol halkaları içeren moleküllerin antimikrobiyal [22,24,25], antiinflamatuvar [26], analjezik [27], antidiyabetik [28], antikanser [29], antidepresan [30] ve antitüberküloz [31] gibi farkl biyolojik aktivitelere sahip olduğu bildirilmiştir. Bu sebepten dolayıdır ki pirazol halkalarına artan ilgi son zamanlarda pirazol halkaları içeren yeni boyarmaddelerin sentezini beraberinde getirmiştir. Bu maksatla çalışma grubumuz tarafından, anilin ve $p$-sübstitüe anilin türevleri kullanılarak, aminopirazol bazlı beş tane yeni disazo boyarmaddeleri sentezlenmiş ve karakterizasyonları yapılarak tekstil literatürüne katılmıştır [32].

$\mathrm{Bu}$ çalışmada ise, grubumuz tarafından yapılan çalışmanın [32] bir devamı olarak, $o$ - ve $m$ - sübstitüe anilin türevleri kullanılarak 3-amino-5-hidroksi-1H-pirazol'den türetilen sekiz tane yeni disazo bileşiğinin sentezi ve bazı spektroskopik özellikleri detaylı olarak sunulmuştur.

\section{Materyal ve Metot}

\subsection{Kullanılan Kimyasal Maddeler ve Cihazlar}

Boyarmaddelerin sentezi, saflaştırılması ve UV-vis çalışmalarında kullanılan kimyasallar Aldrich ve Merck Kimya Şirketlerine aittir ve herhangi bir saflaştırma yapılmadan kullanılmıştır.

IR spektrumları, Perkin Elmer UATR Two Spektrofotometresi ile alınmıştır. ${ }^{1} \mathrm{H}-\mathrm{NMR}$ spektrumları, Agilent 400/55 cihazı ile dimetilsülfoksit (DMSO-d 6 ) içinde referans olarak Tetrametilsilan(TMS) kullanılarak ölçülmüsstür. Boyarmaddelerin erime noktaları Elektrotermal 9100 erime noktası cihazı kullanılarak belirlenmiştir. Boyarmaddelerin element analizi, Leco CHNS-932 analizörü ile gerçekleştirilmiştir. UV-vis spektrumları, Schimadzu UV-1601 spektrofotometresi kullanılarak elde edilmiştir. Absorpsiyon spektrumları $10^{-6} \mathrm{M}$ DMSO, DMF, asetonitril, metanol, asetik asit ve kloroform çözücülerinde alınmıştır. Elde edilen boyarmaddelerin elementel analizleri ve erime noktaları Tablo 1'de verilmiştir.

\subsection{1a-h ve 2a-h boyalarının sentezi}

1a-h ve 2a-h boyarmaddeleri, literatürde verilen prosedür izlenerek sentezlendi $[33,34]$. 1a-h ve 2a-h boyarmaddelerinin sentez şeması Şekil 1'de verilmiştir. 


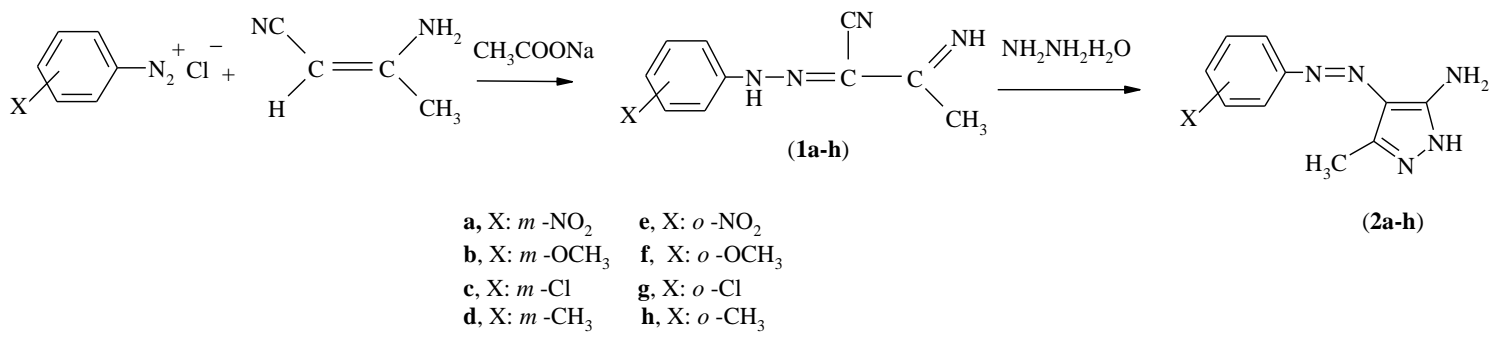

Şekil 1: 1a-h ve 2a-h boyarmaddelerinin sentez şeması

\subsection{Disazo boyarmaddelerin sentezi (3a-h)}

Sentezlenen 2a-h bileşiklerinden $0.01 \mathrm{~mol}$ alınarak bir behere konuldu. Üzerine $10 \mathrm{~mL}$ asetik asit ve $10 \mathrm{~mL}$ hidroklorik asit eklendi. Beher tuz-buz banyosuna konuldu ve çözelti $0-5{ }^{\circ} \mathrm{C}^{\prime}$ ye soğutuldu. Başka bir beherde $10 \mathrm{ml}$ su içinde hazırlanan $\mathrm{NaNO}_{2}$ çözeltisi $(0.69 \mathrm{~g}, 0.01 \mathrm{~mol}), 0-5^{\circ} \mathrm{C}^{\prime}$ de soğutulan bu çözeltiye 1 saat süreyle sürekli karıştırılarak damla damla ilave edildi. Elde edilen diazonyum tuzu çözeltisi, başka bir beherde hazırlanan $\mathrm{KOH}(0.56 \mathrm{~g}, 0.01 \mathrm{~mol})$ ve su $(20 \mathrm{ml})$ içerisinde 3-amino-5hidroksi-1H-pirazol (0.99 g, $0.01 \mathrm{~mol})$ içeren çözeltiye 30 dakika boyunca kısım kısım ilave edildi. $\mathrm{Bu}$ arada sicaklık $0^{-} 5^{\circ} \mathrm{C}$ aralığında, $\mathrm{pH}$ değeri ise sulu sodyum asetat ilavesiyle 7 ile 8 aralığında tutuldu. Karıştırmaya $0-5^{\circ} \mathrm{C}^{\prime}$ de 4 saat devam edildi ve ardından soğuk su $(50 \mathrm{ml})$ ile seyreltildi. Çöken ürünler süzüldü, birkaç kez su (100 $\mathrm{mL}$ ) ile y1kand1, kurutuldu ve DMF- $\mathrm{H}_{2} \mathrm{O}$ 'dan (oran 1: 2) kristallendirilerek 3a boyarmaddesi elde edildi. 3b-h boyarmaddeleri için aynı sentez yöntemi kullanıldı. 3a-h boyarmaddeleri için sentez şeması, Şekil 2'de verilmiştir.
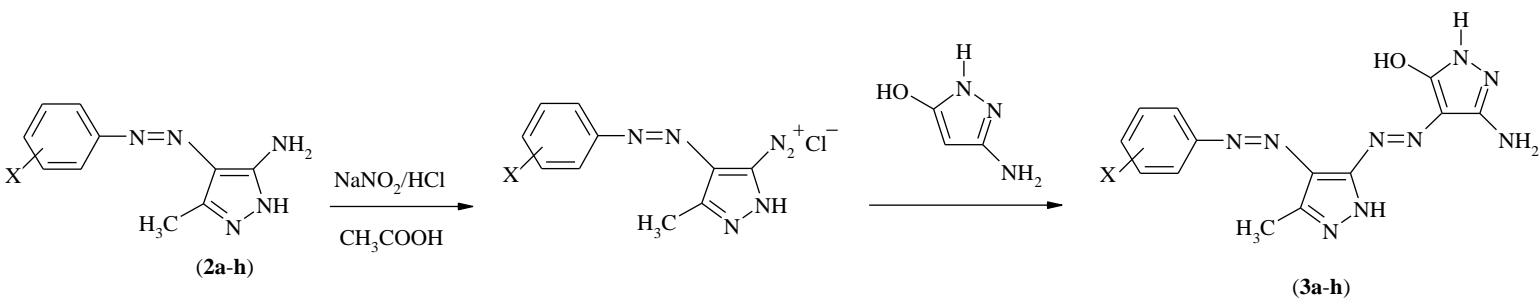

Şekil 2: 3a-h boyarmaddeleri için sentez şeması

\section{Bulgular}

\subsection{Bileşiklerin temel özellikler}

Sentezlenen boyarmaddelerin tamamı amorf yapıda elde edilmiş olup $3 \mathrm{a}$ ve $3 \mathrm{e}$ maddelerinde koyu kahverengi, $3 \mathrm{~b}, 3 \mathrm{~d}$, 3f ve $3 \mathrm{~h}$ maddelerinde kahverengi, $3 \mathrm{c}$ ve $3 \mathrm{~g}$ maddelerinde ise açık kahverengi renkler gözlenmiştir. Sentezlenen boyarmaddelerin verimleri Tablo 1'de verilmiştir. Tablo 1'de görüldüğü gibi en yüksek verimle $3 a$ boyarmaddesi (\% 89) sentezlenirken, 3d boyarmaddesinin verimi (\% 67) en düşük olmuştur. Bilindiği gibi aromatik halkanın aktivitesini azaltan gruplar diazonyum tuzunun pozitif yükünü artırdıkça, diazonyum bileşiğinin reaktivitesi ve ortaya çıkan ürünün verimi artmaktadır. $\mathrm{Bu}$ çalışmada da benzer sonuçlar elde edilmiştir. (Örneğin 3a boyarmaddesi için verim \% 89, 3e boyarmaddesi için verim \% 86) Aromatik halkanın aktivitesini artıran grupları içeren boyarmadde verimleri de düşük bulunmuştur. (Örneğin $3 \mathrm{~b}$ boyarmaddesi için verim \% 72, 3f boyarmaddesi için verim $\%$ 77). 
Tablo 1: 3a-h boyarmaddelerinin element analiz sonuçları

\begin{tabular}{|c|c|c|c|c|c|c|}
\hline \multirow{2}{*}{$\begin{array}{c}\text { Boya } \\
\text { no }\end{array}$} & \multirow{2}{*}{$\begin{array}{l}\text { Molekül } \\
\text { formülü }\end{array}$} & \multirow[t]{2}{*}{ E.N. $\left({ }^{\circ} \mathrm{C}\right)$} & \multirow{2}{*}{$\begin{array}{c}\text { Verim } \\
(\%)\end{array}$} & \multicolumn{3}{|c|}{ Element analiz: Hesaplanan (bulunan) } \\
\hline & & & & $\mathbf{C}$ & $\mathbf{H}$ & $\mathbf{N}$ \\
\hline $\mathbf{3 a}$ & $\mathrm{C}_{13} \mathrm{H}_{12} \mathrm{~N}_{10} \mathrm{O}_{3}$ & $319-320$ & 89 & $43.82(43.64)$ & $3.39(3.46)$ & $39.31(39.12)$ \\
\hline $3 \mathbf{b}$ & $\mathrm{C}_{14} \mathrm{H}_{15} \mathrm{~N}_{9} \mathrm{O}_{2}$ & $285-286$ & 72 & $49.26(49.46)$ & $4.43(4.37)$ & $36.93(36.75)$ \\
\hline $3 c$ & $\mathrm{C}_{13} \mathrm{H}_{12} \mathrm{ClN}_{9} \mathrm{O}$ & $281-282$ & 79 & $45.16(44.93)$ & $3.50(3.41)$ & $36.46(36.67)$ \\
\hline 3d & $\mathrm{C}_{14} \mathrm{H}_{15} \mathrm{~N}_{9} \mathrm{O}$ & 288-289 & 67 & $51.69(51.58)$ & $4.65(4.73)$ & $38.75(38.66)$ \\
\hline $\mathbf{3 e}$ & $\mathrm{C}_{13} \mathrm{H}_{12} \mathrm{~N}_{10} \mathrm{O}_{3}$ & $255-256$ & 86 & $43.82(43.98)$ & $3.39(3.46)$ & $39.31(39.59)$ \\
\hline 3f & $\mathrm{C}_{14} \mathrm{H}_{15} \mathrm{~N}_{9} \mathrm{O}_{2}$ & $211-212$ & 77 & $49.26(49.41)$ & $4.43(4.35)$ & $36.93(37.09)$ \\
\hline $3 g$ & $\mathrm{C}_{13} \mathrm{H}_{12} \mathrm{ClN}_{9} \mathrm{O}$ & $227-228$ & 84 & $45.16(45.38)$ & $3.50(3.61)$ & $36.46(36.29)$ \\
\hline $3 \mathbf{h}$ & $\mathrm{C}_{14} \mathrm{H}_{15} \mathrm{~N}_{9} \mathrm{O}$ & $271-272$ & 71 & $51.69(51.86)$ & $4.65(4.57)$ & $38.75(38.56)$ \\
\hline
\end{tabular}

\subsection{IR spektral çalışmaları ve tautomerizm}

3a-h boyarmaddelerinin Şekil 4'de verildiği gibi 12 olası tautomerik formda mevcut olabileceği düşünülmüştür. 3b bileşiğine ait FT-IR spektrumu Şekil 3'te verilmiştir. 3ah boyarmaddelerinin FT-IR spektrumları incelendiğinde $1674-1629 \mathrm{~cm}^{-1}$ de karbonil bantları $(\mathrm{C}=\mathrm{O})$ görülmüştür. (Tablo 2) Bununla birlikte, FT-IR spektrumlarında hidroksil grubuna ait herhangi bir geniş bant görülmemiştir. $\mathrm{Bu}$ sonuçlar, 3a-h boyarmaddelerinin ağırlıklı olarak kat1 formda T2, T3, T6, T7, T10 veya T11 tautomerik formunda olabileceğini göstermektedir. Daha önce yapılan bazı çalışmalarda da, pirazol boyarmaddelerinin katı formdaki tautomerik yapılarının hidrazo-keto formu lehine olduğu bildirilmiştir. [33, 35, 36].

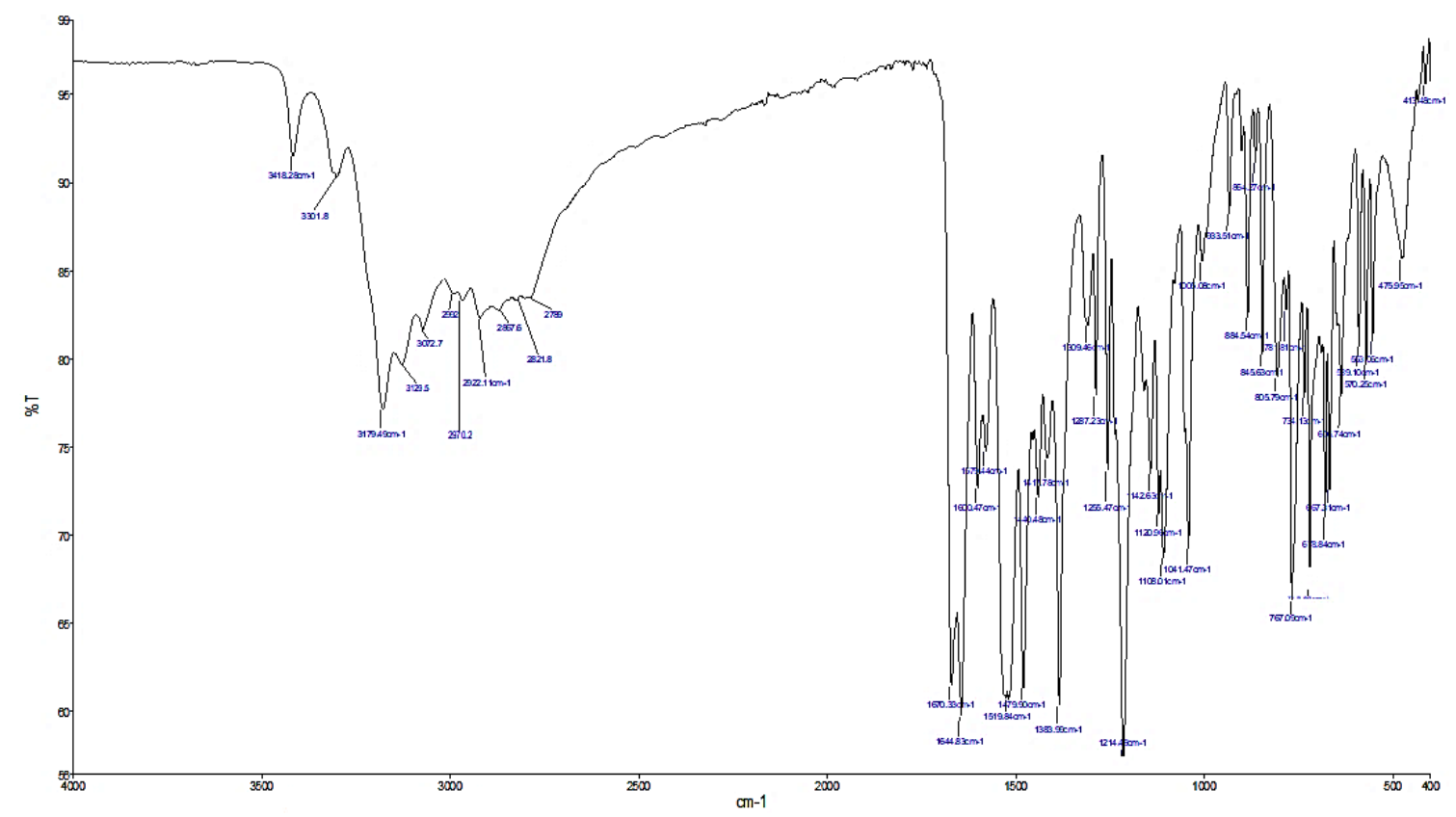

Şekil 3. 3b boyarmaddesinin FT-IR spektrumu 


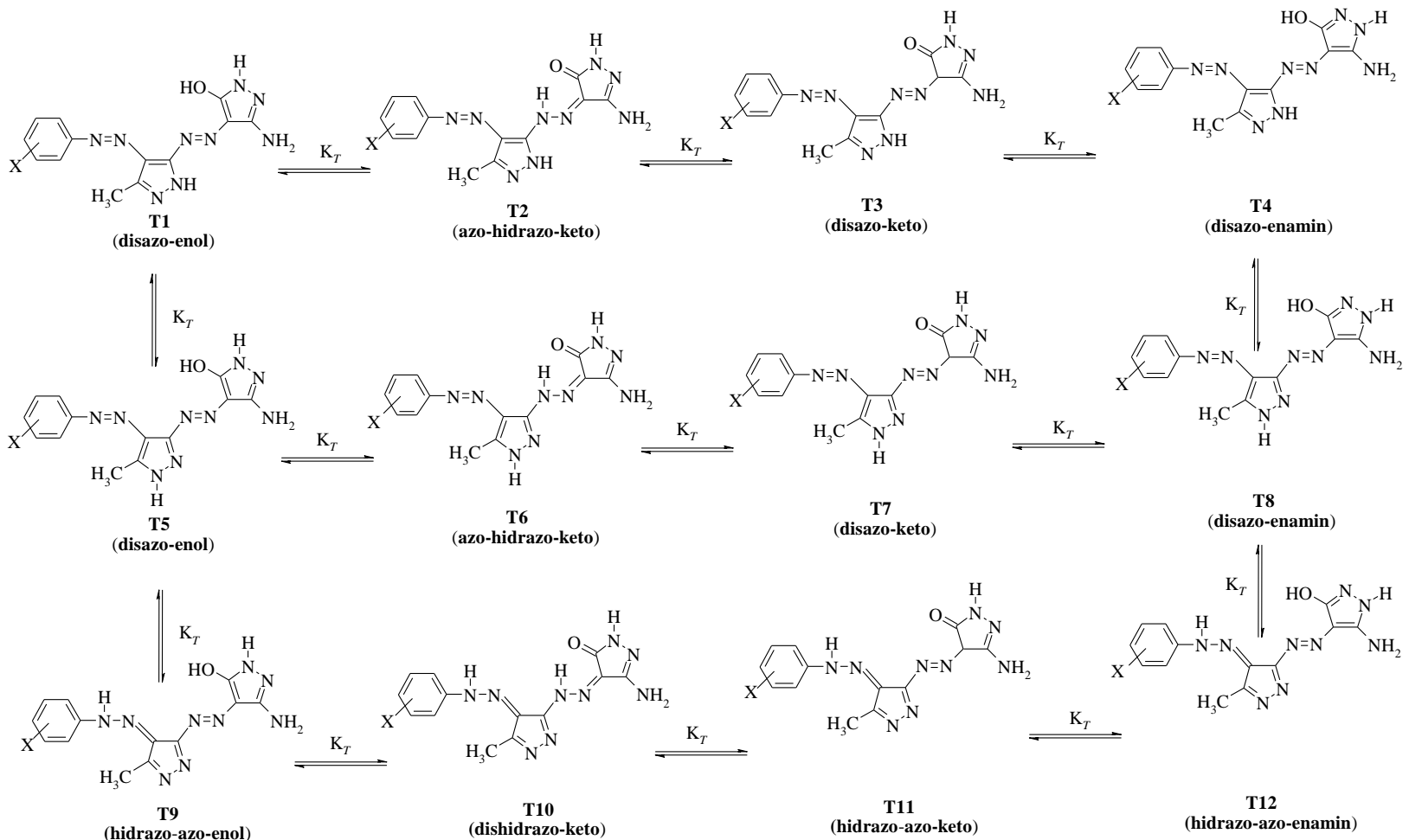

Şekil 4. 3a-h boyarmaddeleri için olası tautomerik formlar

3a-h boyarmaddelerinin FT-IR spektrumları incelendiğinde, amino grubuna $\left(\mathrm{NH}_{2}\right)$ ait $3455-3415 \mathrm{~cm}^{-1}$ ve $3382-3301 \mathrm{~cm}^{-1}$ aralığında iki bant, $3183-3136 \mathrm{~cm}^{-1}$ de NH bantları, $3075-3055 \mathrm{~cm}^{-1}$ de aromatik $\mathrm{CH}$ bantları, 2997-2970 $\mathrm{cm}^{-1}$ 'de alifatik $\mathrm{CH}$ bantları ve 1556-1517 $\mathrm{cm}^{-1}$ de azo $(\mathrm{N}=\mathrm{N})$ bantları da gözlenmiştir. Çalışma grubumuz tarafindan sentezlenen $p$-sübstitüe anilin türevlerinden elde edilen disazo boyarmaddelerinde de benzer sonuçlar elde edilmiştir [32].

\section{3. ${ }^{1} \mathrm{H}$-NMR spektral çalışmaları}

3b bileşiğine ait ${ }^{1} \mathrm{H}$-NMR spektrumu Şekil 5'te verilmiştir. 3a-h boyarmaddelerinin ${ }^{1} \mathrm{H}$ NMR spektrumlarında, sirasiyla 14.05-13.41 ppm (pirazol NH), 13.41-13.00 ppm (pirazol $\mathrm{NH}), 10.73-10.49 \mathrm{ppm}(\mathrm{OH})$ ve 5.80-5.68 ppm $\left(\mathrm{NH}_{2}\right)$ 'de dört geniş pik görülmüştür. Diğer $\delta$ değerleri, Tablo 2'de listelendiği gibi 2.61-2.53 ppm $\left(\mathrm{CH}_{3}\right)$ ve 8.72-6.97 ppm'de (aromatik H) kaydedilmiştir. Bu sonuçlar, boyarmaddelerin DMSO'da T1, T4, T5, T8, T9 ve T12 tautomerik formlarının karışımı halinde bulunabileceğini göstermektedir. Çalışma grubumuz tarafından sentezlenen $p$-sübstitüe anilin türevlerinden elde edilen disazo boyarmaddelerinde de benzer sonuçlar elde edilmiştir [32]. 


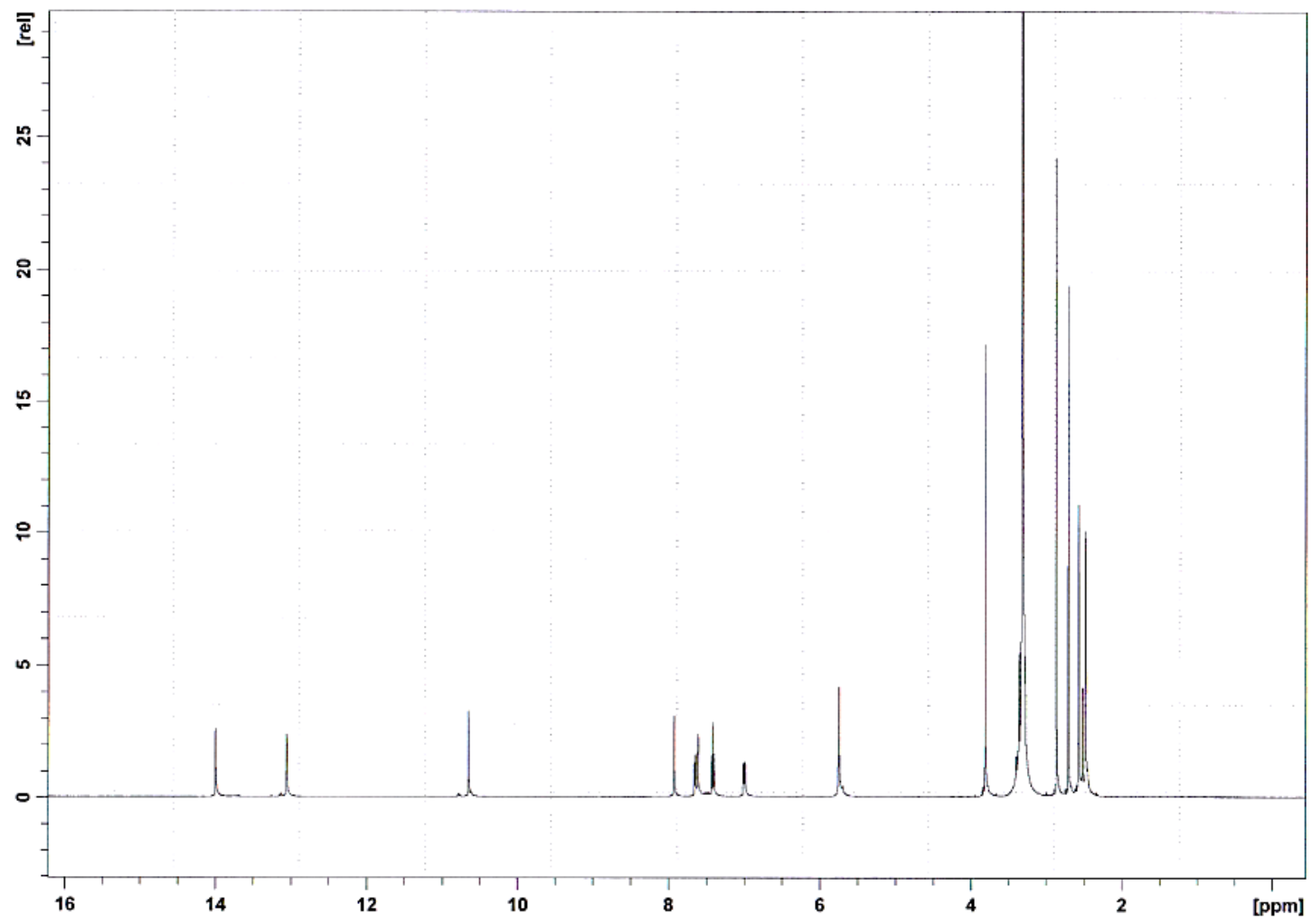

Şekil 5. 3 b boyarmaddesinin ${ }^{1} \mathrm{H}-\mathrm{NMR}$ spektrumu

Tablo 2. 3a-h boyarmaddelerinin FT-IR ve ${ }^{1} \mathrm{H}-\mathrm{NMR}$ sonuçları

\begin{tabular}{|c|c|c|c|c|c|c|c|c|c|}
\hline \multirow{2}{*}{$\begin{array}{l}\text { Boya } \\
\text { no }\end{array}$} & \multicolumn{6}{|c|}{ FT-IR $\left(\mathrm{cm}^{-1}, \mathrm{KBr}\right)$} & \multicolumn{3}{|c|}{${ }^{1}$ H-NMR $(\delta$, ppm, DMSO-d 6$)$} \\
\hline & $v_{\mathrm{NH} 2}$ & $\mathrm{v}_{\mathrm{N}-\mathrm{H}}$ & $v_{\mathrm{Ar}-\mathrm{H}}$ & $v_{\mathrm{Al}-\mathrm{H}}$ & $v_{\mathrm{C}=\mathrm{O}}$ & $v_{\mathrm{N}=\mathrm{N}}$ & Aro.-H & Alif.-H & $\mathbf{X}-\mathbf{H}$ \\
\hline $\mathbf{3 a}$ & $\begin{array}{l}3415, \\
3318\end{array}$ & 3157 & 3072 & 2970 & 1674 & 1519 & $\begin{array}{c}8.72-7.81 \\
(4 \mathrm{H}, \mathrm{m})\end{array}$ & $2.60\left(3 \mathrm{H}, \mathrm{s}, \mathrm{CH}_{3}\right)$ & $\begin{array}{l}14.05(1 \mathrm{H}, \mathrm{g}, \mathrm{NH}) \\
13.22(1 \mathrm{H}, \mathrm{g}, \mathrm{NH}) \\
10.73(1 \mathrm{H}, \mathrm{g}, \mathrm{OH}) \\
5.80\left(2 \mathrm{H}, \mathrm{g}, \mathrm{NH}_{2}\right)\end{array}$ \\
\hline $3 \mathbf{b}$ & $\begin{array}{l}3418 \\
3301\end{array}$ & 3179 & 3072 & 2992 & 1670 & 1519 & $\begin{array}{c}7.94-7.01 \\
(4 \mathrm{H}, \mathrm{m})\end{array}$ & $\begin{array}{c}2.57\left(3 \mathrm{H}, \mathrm{s}, \mathrm{CH}_{3}\right) \\
3.82(3 \mathrm{H}, \mathrm{s} \\
\left.m-\mathrm{OCH}_{3}\right)\end{array}$ & $\begin{array}{l}14.00(1 \mathrm{H}, \mathrm{g}, \mathrm{NH}) \\
13.05(1 \mathrm{H}, \mathrm{g}, \mathrm{NH}) \\
10.65(1 \mathrm{H}, \mathrm{g}, \mathrm{OH}) \\
5.75\left(2 \mathrm{H}, \mathrm{g}, \mathrm{NH}_{2}\right)\end{array}$ \\
\hline $3 c$ & $\begin{array}{l}3419 \\
3321\end{array}$ & 3143 & 3075 & 2989 & 1673 & 1540 & $\begin{array}{c}8.00-7.54 \\
(4 \mathrm{H}, \mathrm{m})\end{array}$ & $2.57\left(3 \mathrm{H}, \mathrm{s}, \mathrm{CH}_{3}\right)$ & $\begin{array}{l}14.01(1 \mathrm{H}, \mathrm{g}, \mathrm{NH}) \\
13.15(1 \mathrm{H}, \mathrm{g}, \mathrm{NH}) \\
10.72(1 \mathrm{H}, \mathrm{g}, \mathrm{OH}) \\
5.78\left(2 \mathrm{H}, \mathrm{g}, \mathrm{NH}_{2}\right)\end{array}$ \\
\hline 3d & $\begin{array}{l}3424 \\
3330\end{array}$ & 3136 & 3070 & 2986 & 1664 & 1540 & $\begin{array}{c}8.14-7.24 \\
(4 \mathrm{H}, \mathrm{m})\end{array}$ & $\begin{array}{c}2.55\left(3 \mathrm{H}, \mathrm{s}, \mathrm{CH}_{3}\right) \\
2.37(3 \mathrm{H}, \mathrm{s}, \\
\left.m-\mathrm{CH}_{3}\right)\end{array}$ & $\begin{array}{l}13.98(1 \mathrm{H}, \mathrm{g}, \mathrm{NH}) \\
13.03(1 \mathrm{H}, \mathrm{g}, \mathrm{NH}) \\
10.65(1 \mathrm{H}, \mathrm{g}, \mathrm{OH}) \\
5.74\left(2 \mathrm{H}, \mathrm{g}, \mathrm{NH}_{2}\right)\end{array}$ \\
\hline $3 e$ & $\begin{array}{l}3446 \\
3338\end{array}$ & 3182 & 3068 & 2984 & 1668 & 1517 & $\begin{array}{c}7.94-7.58 \\
(4 \mathrm{H}, \mathrm{m})\end{array}$ & $2.59\left(3 \mathrm{H}, \mathrm{s}, \mathrm{CH}_{3}\right)$ & $\begin{array}{l}13.78(1 \mathrm{H}, \mathrm{g}, \mathrm{NH}) \\
13.26(1 \mathrm{H}, \mathrm{g}, \mathrm{NH}) \\
10.59(1 \mathrm{H}, \mathrm{g}, \mathrm{OH}) \\
5.78\left(2 \mathrm{H}, \mathrm{g}, \mathrm{NH}_{2}\right)\end{array}$ \\
\hline $3 f$ & $\begin{array}{l}3442 \\
3382\end{array}$ & 3168 & 3055 & 2997 & 1629 & 1556 & $\begin{array}{c}7.93-6.97 \\
(4 \mathrm{H}, \mathrm{m})\end{array}$ & $\begin{array}{c}2.53\left(3 \mathrm{H}, \mathrm{s}, \mathrm{CH}_{3}\right) \\
3.85(3 \mathrm{H}, \mathrm{s} \\
\left.o-\mathrm{OCH}_{3}\right)\end{array}$ & $\begin{array}{l}13.41(1 \mathrm{H}, \mathrm{g}, \mathrm{NH}) \\
13.00(1 \mathrm{H}, \mathrm{g}, \mathrm{NH}) \\
10.49(1 \mathrm{H}, \mathrm{g}, \mathrm{OH}) \\
5.68\left(2 \mathrm{H}, \mathrm{g}, \mathrm{NH}_{2}\right)\end{array}$ \\
\hline $3 g$ & $\begin{array}{l}3421 \\
3341\end{array}$ & 3169 & 3063 & 2986 & 1672 & 1539 & $\begin{array}{c}7.58-7.12 \\
(4 \mathrm{H}, \mathrm{m})\end{array}$ & $2.56\left(3 \mathrm{H}, \mathrm{s}, \mathrm{CH}_{3}\right)$ & $\begin{array}{l}13.76(1 \mathrm{H}, \mathrm{g}, \mathrm{NH}) \\
13.41(1 \mathrm{H}, \mathrm{g}, \mathrm{NH}) \\
10.56(1 \mathrm{H}, \mathrm{g}, \mathrm{OH}) \\
5.76\left(2 \mathrm{H}, \mathrm{g}, \mathrm{NH}_{2}\right)\end{array}$ \\
\hline $3 \mathbf{h}$ & $\begin{array}{l}3455 \\
3326\end{array}$ & 3183 & 3059 & 2991 & 1674 & 1538 & $\begin{array}{c}7.41-7.29 \\
(4 \mathrm{H}, \mathrm{m})\end{array}$ & $\begin{array}{c}2.61\left(3 \mathrm{H}, \mathrm{s}, \mathrm{CH}_{3}\right) \\
2.34(3 \mathrm{H}, \mathrm{s}, \\
\left.o-\mathrm{CH}_{3}\right)\end{array}$ & $\begin{array}{l}13.57(1 \mathrm{H}, \mathrm{g}, \mathrm{NH}) \\
13.07(1 \mathrm{H}, \mathrm{g}, \mathrm{NH}) \\
10.57(1 \mathrm{H}, \mathrm{g}, \mathrm{OH}) \\
5.75\left(2 \mathrm{H}, \mathrm{g}, \mathrm{NH}_{2}\right)\end{array}$ \\
\hline
\end{tabular}




\subsection{UV-vis absorpsiyon çalışmaları(çözüicü etkileri)}

Boyarmaddelerin absorpsiyon spektrumları farklı çözücüler $\left(10^{-6} \mathrm{M}\right)$ kullanılarak 350$700 \mathrm{~nm}$ dalga boyu aralığında kaydedilmiştir. 3a-h boyarmaddelerinin maksimum dalga boyu değerlerinin değişimi Tablo 3 'de verilmiştir. Tablo 3 incelendiğinde tüm boyarmaddelerin DMF içinde, bir maksimum absorpsiyon değeri ile bir omuz verdiği görülür. $\mathrm{Bu}$ durum $\mathrm{DMF}$ içinde tüm boyarmaddelerin Şekil 4'de verilen tautomerik formların bir karışımı halinde bulunabileceğini düşündürmüştür.

Öte yandan, 3a-h boyarmaddelerinin diğer çözücülerde (DMSO, asetonitril, metanol, asetik asit ve kloroform) tek bir maksimum absorpsiyon piki gösterdiği gözlenmiştir. Bundan dolayı bahsi geçen çözücülerde 3a-h boyarmaddelerinin tek bir tautomerik formda bulunabileceği düşünülmektedir.

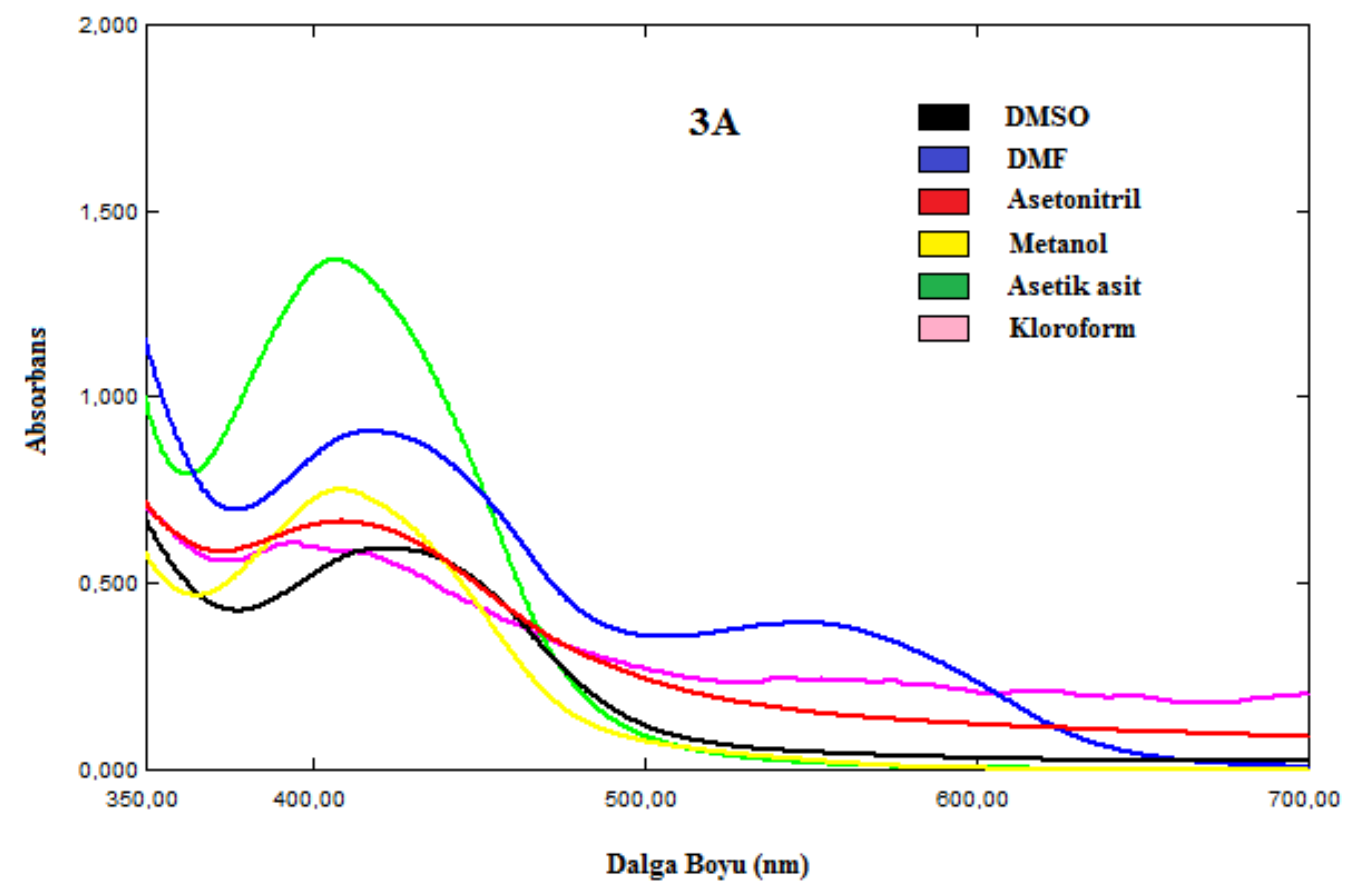

Şekil 6. 3a boyarmaddesinin farklı çözücüler içindeki absorpsiyon spektrumu

3a-h boyarmaddelerinin maksimum absorpsiyon değerleri, kloroform ile karşılaştırıldığında DMF ve DMSO içinde batokromik kaymalar görülürken, diğer çözücülerde belirgin bir değişiklik gözlenmemiştir. Örnek olarak, 3a için, DMSO'da $\lambda$ maks değeri $423 \mathrm{~nm}$ iken, DMF'de $417 \mathrm{~nm}$ (tepe) ve $549 \mathrm{~nm}$ (omuz), kloroformda 410 $\mathrm{nm}$ ve diğer çözeltilerde $405 \mathrm{~nm}$ ile $409 \mathrm{~nm}$ arasındadır (Şekil 6). DMSO ve DMF'nin dielektrik sabiti diğer çözücülerinkinden daha yüksek olduğu için boyarmaddelerin bu çözücülerde batokromik bir kayma göstermesi beklenen bir sonuçtur. Öte yandan boyarmaddelerin asetonitril, metanol, asetik asit ve kloroform içerisindeki absorpsiyon değerleri arasında önemli bir fark bulunmamıştır. Örneğin, 3a için, $\lambda$ maks değeri asetonitrilde $409 \mathrm{~nm}$, kloroformda $410 \mathrm{~nm}$, metanolde $408 \mathrm{~nm}$ ve asetik asitte $405 \mathrm{~nm}$ bulunmuştur. 
Tablo 3: 3a-h boyarmaddelerinin $\lambda$ maks(nm) değerleri üzerine çözücü etkisi

\begin{tabular}{ccccccc}
\hline $\begin{array}{c}\text { Boya } \\
\text { no }\end{array}$ & DMSO & DMF & Asetonitril & Metanol & Asetik asit & Kloroform \\
\hline 3a & 423 & 417,549 o & 409 & 408 & 405 & 410 \\
3b & 415 & 410,528 o & 405 & 408 & 407 & 405 \\
3c & 416 & 413,526 o & 406 & 405 & 406 & 405 \\
3d & 413 & 409,528 o & 403 & 405 & 406 & 404 \\
3e & 418 & 414,547 o & 406 & 406 & 405 & 406 \\
3f & 409 & 406,523 o & 401 & 405 & 403 & 402 \\
3g & 416 & 421,534 o & 397 & 402 & 397 & 394 \\
3h & 405 & 402,521 o & 397 & 398 & 399 & 397 \\
\hline
\end{tabular}

\section{5. $U V$-vis (asit ve baz etkisi)}

Metanol içindeki her boyarmadde çözeltisine $0.1 \mathrm{M} \mathrm{HCl}$ ve $0.1 \mathrm{M} \mathrm{KOH}$ eklenerek elde edilen absorpsiyon spektrumları, sadece metanol içeren boyarmadde çözeltilerininki ile karşılaştırılmıştır. Metanol içindeki boyarmaddelerin absorpsiyon spektrumlarının baz ilavesine çok duyarlı olduğu görülmüştür. Tablo 4, boyarmaddelere $\mathrm{KOH}$ ilavesinin $\lambda$ maks değerlerini batokromik olarak kaydırdığını göstermektedir. Örneğin, 3a için, $\lambda$ maks değerleri metanolde $408 \mathrm{~nm}$ ve bazik metanolde $484 \mathrm{~nm}$ bulundu (Şekil 7). Bu sonuç, bazik metanol içindeki boyarmaddelerin tautomerik yapılarının metanol içindeki tautomerik yapılardan farklı olabileceğini göstermiştir. Tüm boyarmaddelerin bazik metanolde ortak bir anyonik formda bulunabileceğini söylemek mümkündür.

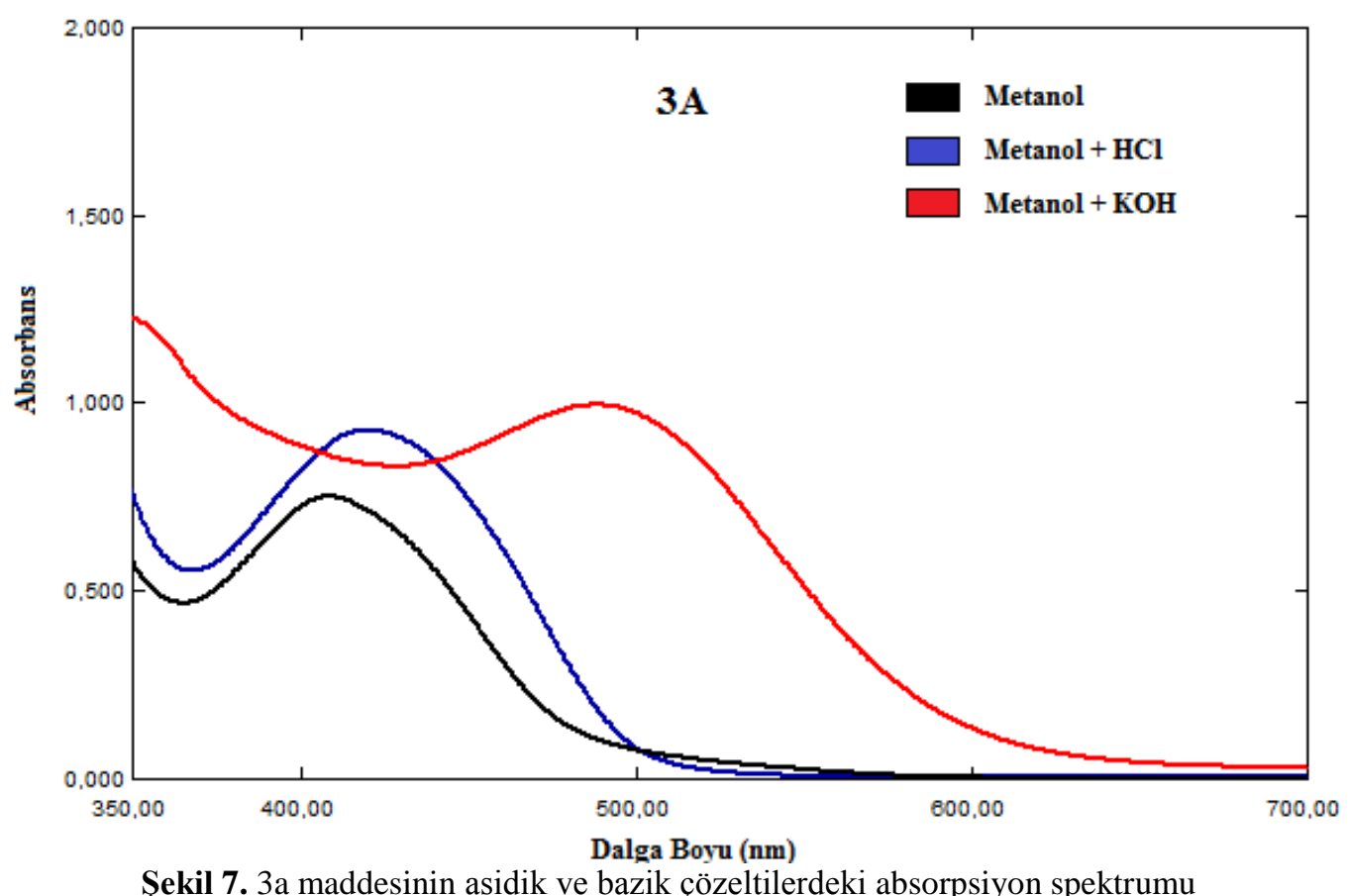

Metanol içindeki boyarmadde çözeltilerine $0.1 \mathrm{M} \mathrm{HCl}$ ilave edildiğinde benzer bir sonuç elde edilmiştir. Metanol içindeki boyarmadde çözeltisine asit eklenmesi, $\lambda$ maks değerlerini (metanoldekine göre) hafifçe değiştirmiştir. Örneğin, $3 \mathrm{~g}$ için, $\lambda$ maks değeri metanolde $402 \mathrm{~nm}$ ve asidik metanolde $400 \mathrm{~nm}$ olarak bulunmuştur. Bu durum, bütün boyarmaddelerin asidik metanol ortamında ayrışmamış bir durumda olabileceğini düşündürmüştür. 
Tablo 4: 3a-h boyarmaddelerinin asidik ve bazik çözeltilerde maksimum absorpsiyon değerleri

\begin{tabular}{cccc}
\hline & \multicolumn{3}{c}{$\boldsymbol{\lambda}_{\text {maks }}(\mathbf{n m})$} \\
\cline { 2 - 4 } Boya no & Metanol & Metanol + KOH & Metanol + HCl \\
\hline 3a & 408 & 484 & 415 \\
3b & 408 & 474 & 417 \\
3c & 405 & 479 & 418 \\
3d & 405 & 471 & 418 \\
3e & 406 & 487 & 416 \\
3f & 405 & 492 & 416 \\
3g & 402 & 494 & 400 \\
3h & 398 & 465 & 412 \\
\hline
\end{tabular}

\section{Sonuç ve Yorum}

Bu çalışmada 8 tane yeni disazo boyarmaddesi sentezlenmiştir. Boyarmaddeler için on iki farklı tautomerik yapı önerilmiştir. FT-IR sonuçları, katı boyarmaddelerin keto tautomerik formda (T2, T3, T6, T7, T10 veya T11) olabileceğini gösterirken, ${ }^{1} \mathrm{H}-\mathrm{NMR}$ sonuçları boyarmaddelerin DMSO içinde enol tautomerik formunda (T1, T4, T5, T8, T9 ve T12) olabileceğini göstermiştir.

UV-vis çalışmaları sentezlenen tüm boyarmaddelerin DMF'de Şekil 4'te gösterilen tautomerik formların bir karışımı olarak mevcut olabileceğini, diğer çözücülerde ise tek bir tautomerik formda bulunabileceğini düşündürmüştür. Metanol içindeki tüm boyarmaddelerin absorpsiyon spektrumlarının alkali ilavesine $(0.1 \mathrm{M} \mathrm{KOH})$ oldukça duyarlı olduğu görülmüş ve baz ilavesiyle batokromik kaymalar gözlenmiştir.

Sentezlenilen ve karakterize edilen bu 8 yeni disazo boyarmadde ile tekstil ve boya literatürüne yeni boyarmaddeler eklendi. Bu yeni bileşiklerin sentezi ile literatürde azo boyarmaddelerin çeşitliliğinin ve kullanım alanlarının artması amaçlandı.

\section{Araştırmacıların Katkı Oranı Beyanı}

Fati YILDIRIM: Araştırma, Orijinal Taslak Yazımı, İnceleme ve Düzenleme

\section{Destek ve Teşekkür Beyanı}

$\mathrm{Bu}$ çalışma Pamukkale Üniversitesi Bilimsel Araştırma Projesi Koordinasyon Birimi tarafından kabul edilen 2016 HZDP 036 numaralı proje kapsamında gerçekleştirilmiştir. Pamukkale Üniversitesi ve Bilimsel Araştırma Projesi Koordinasyon Birimine teşekkür ederim.

\section{Çatışma Beyanı}

Bu çalışmanın yazarı olarak herhangi biriyle bir çatışma beyanımın olmadığını bildiririm.

\section{Etik Kurul Onayı ve Aydınlatılmış Onam Bilgileri}

$\mathrm{Bu}$ çalışmanın yazarı olarak herhangi bir etik kurul onayı ve aydınlatılmış onam bilgileri beyanımın olmadığını bildiririm.

\section{Kaynakça}

[1] J. F. Dawson, “Developments in disperse dyes,'” Color. Technol., 9, 25-35, 1978.

[2] H. Zollinger, "Color Chemistry: Synthesis, Properties and Application of Organic Dyes and Pigments,' third ed. Wiley-VCH, Weinheim, 161-167, 2003.

[3] H. Yousefi, A. Yahyazadeh, E. O. Moradi Rufchahi, M. Rassa, "Synthesis, spectral properties, biological activity and application of new 4-(benzyloxy)phenol derived azo dyes for polyester fiber dyeing,' J. Mol. Liq., 180, 51-58, 2013.

[4] H. F. Rizk, S. A. Ibrahim, M. A. El-Borani, "Synthesis, fastness properties, color assessment and antimicrobial activity of some azo reactive dyes having pyrazole moiety,' Dyes and Pigments, 112, 86-92, 2015.

[5] L. He, L. Lu, S. Zhang, H. S. Freeman, "Synthesis and application of yellow azoanthraquinone disperse dyes for polylactide fibres,' Color. Technol., 126(2), 92-96, 2010. 
[6] M. A. Metwally, E. Abdel-latif, F. A. Amer, G. Kaupp, "Synthesis of new 5-thiazolyl azo-disperse dyes for dyeing polyester fabrics,"' Dyes and Pigments, 60(3), 249-264, 2004.

[7] N. M. Parekh, S. K. Sahoo, K. C. Maheria, "Quantum chemical studies and dyeing performance of some novel benzoquinoline based heterocyclic monoazo dyes on polyester fiber,' Dyes and Pigments, 95(1), 142-148, 2012.

[8] H. Z. Shams, M. H. Helal, F. Mohamed, S. A. Abd-Elhafiz, "A novel synthesis of 1-oxo-thieno [3', 2'-3, 4] pyrazolo [1, 2-a] pyrazole azo dye systems for dyeing of synthetic and modified cellulose fibers,' Pigment Resin Technol., 30(3), 158-167, 2001.

[9] F. Karc1, A. Demirçalı, F. Karcı, I. Kara, F. Ucun, “The synthesis, spectroscopic properties and theoretical calculation of some novel disperse disazo dyes derived from 3-amino-5-hydroxy-1phenylpyrazole,' J. Mol. Struct., 935, 19-26, 2009.

[10] J. H. Choi, J. Y. Choi, Y. Lee, A. D. Towns, C. Yoon, "Novel azo dyes derived from phthalimide. Part 2: dyeing properties and colour fastness on polyester fibres,' Color. Technol., 124(6), 364-369, 2008.

[11] A. Z. Sayed, M. S. Aboul-Fetouh, H. S. Nassar, "Synthesis, biological activity and dyeing performance of some novel azo disperse dyes incorporating pyrazolo [1,5-a] pyrimidines for dyeing of polyester fabrics,' J. Mol. Struct., 1010, 146-151, 2012.

[12] G. H. Elgemeie, M. H. Helal, H. M. El-Sayed, "Recent trends in synthesis and application of nitrogen heterocyclic azo dyes," Pigment Resin Technol., 30(4), 210-228, 2001.

[13] J. Mikroyannidis, D. Tsagkournos, P. Balraju, G. Sharma, "Low band gap dyes based on 2-styryl-5phenylazo-pyrrole: synthesis and application for efficient dyesensitized solar cells," J. Power Sources, 196, 4152-4161, 2011.

[14] F. Ruyffelaere, V. Nardello, R. Schmidt, J. M. Aubry, "Photosensitizing properties and reactivity of aryl azo naphtol dyes towards singlet oxygen,' J Photochem Photobiol A: Chem., 183, 98-105, 2006.

[15] O. A. Adegoke, T. E. Adesuji, O. E. Thomas, "Novel colorimetric sensors for cyanide based on azohydrazone tautomeric skeletons,', Spectrochim Acta A Mol Biomol Spectrosc., 128, 147-152, 2014.

[16] P. J. Coelho, L. M. Carvalho, A. M. C. Fonseca, M. M. M. Raposo, "Photochromic properties of thienylpyrrole azo dyes in solution,' Tetrahedron Lett., 47, 3711-3714, 2006.

[17] E. Pevzner, B. Ehrenberg, "Principal component analysis of the absorption and resonance Raman spectra of the metallochromic indicator antipyrylazo III," Spectrochim Acta A Mol Biomol Spectrosc., 56, 637-651, 2000.

[18] A. Y. Al-Ahmad, Q. M. A. Hassan, H. A. Badran, K. A. Hussain, "Investigating some linear and nonlinear optical properties of the azo dye (1-amino-2-hydroxy naphthalin sulfonic acid-[3-(4-azo)]4-amino diphenyl sulfone)," Opt Laser Technol., 44(5), 1450-1455, 2012.

[19] M. Erfantalab, H. Khanmohammadi, "New 1, 2, 4-triazole-based azo-azomethine dye. Part III: synthesis, characterization, thermal property, spectrophotometric and computational studies," Spectrochim Acta A. Mol Biomol Spectrosc., 125, 345-352, 2014.

[20] F. Yıldırım, A. Demirçalı, F. Karcı, A. Bayrakdar, P. T. Taşlı, H. H. Kart, "New coumarin-based disperse disazo dyes: Synthesis, spectroscopic properties and theoretical calculations,' J. Mol Struc., 223, 557-565, 2016.

[21] M. Yazdanbakhsh, H. Yousefi, M. Mamaghani, E. Moradi, M. Rassa, H. Pouramir, "Synthesis, spectral characterization and antimicrobial activity of some new azo dyes derived from 4, 6dihydroxypyrimidine," J. Mol. Liq., 169, 21-26, 2012.

[22] F. Karc1, N. Şener, M. Yamaç, İ. Şener, A. Demirçalı, "The synthesis, antimicrobial activity and absorption characteristics of some novel heterocyclic disazo dyes," Dyes and Pigments, 80, 47-52, 2009.

[23] H. Xu, X. Zeng, "Synthesis of diaryl-azo derivatives as potential antifungal agents,' Bioorg Med Chem Lett., 20(14), 4193-4195, 2010.

[24] T. I. El-Emary, S. A. A. El-Mohsen, "Multi-component one-pot synthesis and antimicrobial activities of 3-Methyl-1,4-diphenyl-7-thioxo-4, 6, 8, 9-tetrahydro-pyrazolo[5, 4-b] pyrimidino [5, 4e] pyridine-5-one and related derivatives,"' Molecules, 17, 14464-14483, 2012

[25] A. P. Acharya, R. D. Kamble, S. V. Hese, S. N. Kadam, R. N. Gacche, B. S. Dawane, "Eco-friendly synthesis of novel indeno-pyrazole derivatives and their in-vitro antimicrobial screening," Org. Commun, 7(2), 68-76, 2014.

[26] S. G. Alegaon, K. R. Alagawadi, M. Garg, K. Dushyant, D. Vinod, “'1, 3, 4-Trisubstituted pyrazole analogues as promising anti-inflammatory agents,' Bioorg Chem., 54, 51-59, 2014.

[27] P. D. Gokulan, B. Jayakar, V. Alagarsamy, V. R. Solomon, "Synthesis and Pharmacological Investigation of 5-Substituted-3-Methylsulfanyl-1H-Pyrazole-4-Carboxylic Acid Ethyl Esters as New Analgesic and anti-Inflammatory Agents,'” Arzneimittelforschung, 62, 457-462, 2012. 
[28] H. M. Faidallah, M. M. Al-Mohammadi, K. A. Alamry, K. A. Khan, "Synthesis and Biological Evaluation of Fluoropyrazolesulfonylurea and Thiourea Derivatives as Possible AntidiabeticAgents,', J. Enzyme Inhib. Med. Chem., 31, 157-163, 2016.

[29] A.R. Ali, E.R. El-Bendary, M. A. Ghaly, I. A. Shehata, "Synthesis, in vitro anticancer evaluation and in silico studies of novel imidazo [2, 1-b] thiazole derivatives bearing pyrazole moieties,' Eur J Med Chem., 75, 492-500, 2014.

[30] M. Abdel-Aziz, GE-DA Abuo-Rahma, AA Hassan, "Synthesis of novel pyrazole derivatives and evaluation of their antidepressant and anticonvulsant activities," Eur J Med Chem., 44, 3480-3487, 2009

[31] V. Pathak, H. K. Maurya, S. Sharma, K. K. Srivastava, A. Gupta, "Synthesis and biological evaluation of substituted 4, 6-diarylpyrimidines and 3, 5-diphenyl-4, 5-dihydro-1H-pyrazoles as antitubercular agents,' Bioorg Med Chem Lett., 24, 2892-2896, 2014

[32] F. Yıldırım, A. Demirçalı, P. T. Taşl, F. Karcı, "New disazo dyes derived from aminopyrazoles: synthesis, spectroscopic properties, computational study and structural properties" Coloration Technology, baskida, 2021.

[33] M. H. Elnagdi, M. M. M. Sallam, H. M. Fahmy, S. A. M. Ibrahim, M. A. M. Elias, "Reactions with the Arylhydrazones of $\alpha$-Cyanoketones: The Structure of 2-Arylhydrazono-3-ketimino-nitriles,' Helv Chim.Acta., 59(2), 551-557, 1976.

[34] M. H. Elnagdi, G. E. H. Elgemeie, F. A. E. Abd-Ellal, "Recent Developments in the Synthesis of Pyrazole Derivative,' Heterocycles, 23(12), 3121- 3153, 1985.

[35] F. Karc1, "Synthesis of disazo dyes derived from heterocyclic components,' Color Technol., 121, 275-280, 2005.

[36] F. Karc1, A. Demirçalı, "Synthesis of disazo pyrazolo[1,5-a]pyrimidines,'” Dyes and Pigments, 74, 288-297, 2007. 